\title{
Maximum energy in electromagnetic module volume
}

\author{
V.Yu. Neyman \\ Novosibirsk State Technical University \\ Novosibirsk, Russian Federation \\ E-mail: nv.nstu@ngs.ru
}

\begin{abstract}
The relation between maximum mechanical energy and electromagnetic module volume for single acceleration of the electromagnetic device armature in longitudinal magnetic field has been analytically established. Mathematical formulation of kinetic energy and mechanical work and the Maxwell equation for electromagnetic force have been used. The influence of magnetic field in the operating air gap on mechanical work and kinetic energy values has been shown. The electromagnetic module volume maximum energy dependences on magnetic field change and initial armature penetration have been derived.
\end{abstract}

Keywords - electromagnetic module; maximum energy; kinetic energy; electromagnetic force; Maxwell equation; mechanical work.

\section{INTRODUCTION}

The development of electromagnetic devices based on pulse linear electromagnetic motors (LEMM) has led to increase of their specific energy parameters in several times [1-6]. For example, such integrated indicator of design improvement as impact energy per motor unit mass has been reduced to $6.4 \mathrm{~J} / \mathrm{kg}$ [7].

The analysis of the best shock machines operating modes shows that impact energy per motor unit mass belongs to the interval $0.83 \ldots 5.56 \mathrm{~J} / \mathrm{kg}$ for unit impact energy $0.2 \ldots 400000$ J. The maximal running-out speed of the armature achieves 3 .. $5.5 \mathrm{~m} / \mathrm{s}$. The need and tendency of the further growth of parameters represent practical interest for definition of their maximal values.

Generally the problem of the further research can be formulated as follows: it is necessary to define the maximal values of specific impact energy and speed of mechanical movement at single acceleration of the electromagnetic device armature in longitudinal magnetic field.

\section{Method OF ANALYSIS}

The LEMM armature single accelerated motion in a longitudinal magnetic field is considered.

Movement of the armature under the action of electromagnetic force, as a rule, is connected with producing of mechanical work:

$$
A_{\text {mec }}=\frac{m_{\mathrm{a}} v^{2}}{2}
$$

where $m_{\mathrm{a}}$ is the armature weight, $v$ is the armature mechanical motion linear speed. The mechanical work in case of counteracting force absence completely converses into kinetic energy.

On the other hand, LEMM mechanical work is:

$$
A_{\mathrm{mec}}=\int_{x_{0}=0}^{x} F_{\mathrm{em}} d x
$$

where $x_{0}$ and $x$ are the initial and current coordinates of an armature position $x=\delta_{\max }\left(1-k_{\mathrm{a}}\right) \quad$ (Fig.1), $\quad F_{\mathrm{em}}$ is the electromagnetic force:

$$
F_{\mathrm{em}}=\left.\left(\frac{i^{2}}{2} \frac{d L}{d x}\right)\right|_{i=\mathrm{const}}=\frac{B^{2} S}{2 \mathrm{~m}_{0}},
$$

where $B$ is the magnetic induction in the operating air gap, $S$ is the area of armature section, $\mathrm{M}_{0}$ is the air magnetic permeability.

According to available recommendations, the change of induction can be presented in the following form:

$$
B=B_{\max }\left(\frac{x}{a_{\max }}\right)^{n},
$$

where $B_{\max }$ is the maximal saturation in the operating air gap, $\triangle_{\max }$ is the operating air gap size corresponding to the maximum armature displacement.

When the armature is moving, the induction change in the operating air gap $\delta=\delta_{\max }\left(1-k_{\mathrm{a}}\right)-x$ is determined by the index of power $n$. If $n=0$, then the induction in the operating air gap is maximum $B=B_{\max }$ in spite of the armature coordinate. If $n=1$, then the induction linearly changes.

The relative induction $B^{*}=f x^{*}, n$ as the function of the armature coordinate and power index $n$ for the penetration 
coefficient $k_{\mathrm{a}}=0$ is plotted in Fig. 2, where $B^{*}=B / B_{\max }$ is the relative induction, $x^{*}=x / \delta_{\max }$ is the relative displacement.

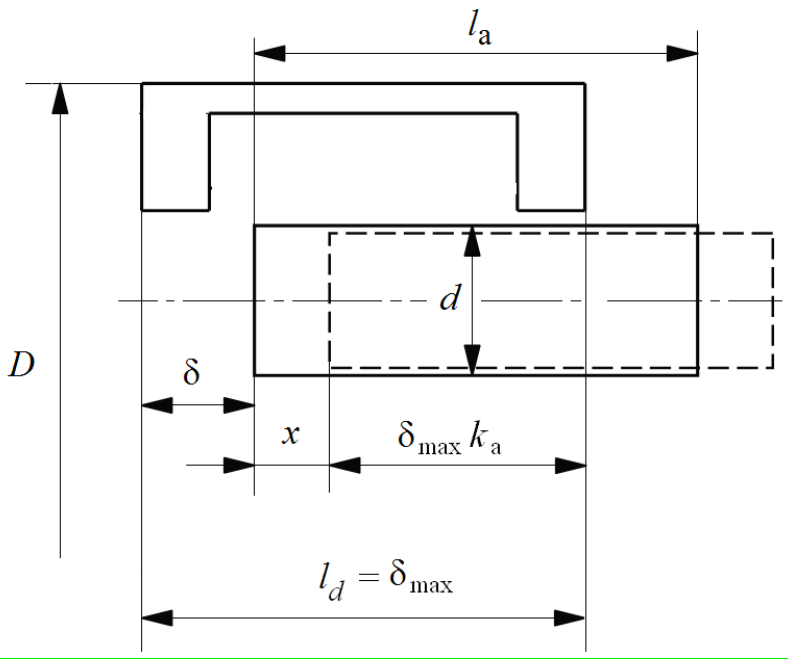

Fig.1. The LEMM arrangement

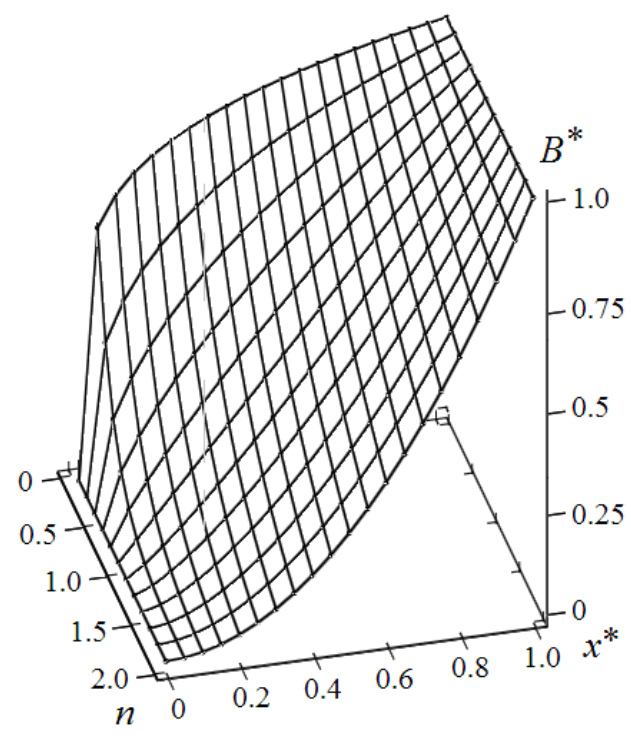

Fig. 2. The plot of $B^{*}=f x^{*}, n$.

The degree of the magnetic flux increase is defined by the parameter $n$. The obtained induction change dependences on armature position coordinate for $0<n<1$ are similar to real. The value $n=0$ corresponds to the maximal magnetic flux increase and constant magnetic induction $B$ at the whole armature motion interval.
For $n=1$ the induction change in the operating gap is characterized by linear dependence. With growth of the parameter $n$ the induction speed change noticeably decreases.

As a result of substitution (3) and (4) in (2) expression for mechanical work takes the form:

$$
A_{\mathrm{mec}}=\int_{x_{0}=0}^{x} \frac{B_{\mathrm{max}}^{2} S}{2 \mathrm{M}_{0}}\left(\frac{x}{\delta_{\max }}\right)^{2 n} d x
$$

After integration (5), taking into account of $x$ limits, the expression (5) becomes:

$$
A_{\mathrm{mec}}=\left.\frac{B_{\max }^{2} S x^{2 n+1}}{2 \mathrm{M}_{0} \text { म }_{\max }^{2 n}(2 n+1)}\right|_{x_{0}=0} ^{x=\text { д }}=\frac{B_{\max }^{2} S \text { д }^{2 n+1}}{2 \mathrm{M}_{0} \text { म }_{\max }^{2 n}(2 n+1)} .
$$

As a rule, the displacement of the LEMM armature takes place within the limits of the coil length.

The size of the operating air gap д is expressed through the relative armature initial displacement $x^{*}$ and the coil linear size $l_{\mathrm{a}}$. If the armature displacement is equal to the coil length $\left(д_{\max }=l_{\mathrm{a}}\right)$, then $д=д_{\max }\left(1-k_{\mathrm{a}}\right)$. Therefore the mechanical work (6) takes the form:

$$
A_{\mathrm{mec}}=\frac{B_{\max }^{2} S \text { म }_{\max }\left(1-k_{\mathrm{a}}\right)^{2 n+1}}{2 \mathrm{M}_{0}(2 n+1)} .
$$

The size $S д_{\max }=V_{\mathrm{a}}^{l}$ in (7) at ${д_{\max }}=l_{\mathrm{a}}$ characterizes the volume of the operating air gap superseded by the armature. Limits of change of the displacement factor are $k_{\mathrm{a}}=0 \ldots 1.0$. At $k_{\mathrm{a}}=0$, the initial coordinate of the armature position corresponds to the operating air gap $д=Д_{\max }$.

At $k_{\mathrm{a}}=1$ and д $=0$, the armature is completely involved and the magnetic system is balanced and work is not made.

With respect to (7) the armature moves within the limits of the length $l_{\mathrm{a}}$. Expressing the maximal armature displacement with respect to its linear size $l_{\mathrm{a}}$ through the relative initial penetration depth $k_{\mathrm{a}}$ gives:

$$
A_{\mathrm{mec}}=\frac{B^{2} S}{2 \mathrm{~m}_{0}} l_{\mathrm{a}} \frac{1-k_{\mathrm{a}}^{2 n+1}}{(2 n+1)} .
$$

The size $S l_{\mathrm{a}}=V_{\mathrm{a}}^{l}$ in (8) defines the volume of the operating air gap superseded by the armature at its full penetration (д $=0$ ).

Expression for mechanical work (8) can be reduced to the form: 


$$
A_{\mathrm{mec}}=\frac{B^{2} V_{\mathrm{a}}^{l} 1-k_{\mathrm{a}}^{2 n+1}}{2 \mathrm{M}_{0}(2 n+1)} .
$$

From the condition of magnetic circuit equal loading the volume $V_{\mathrm{a}}^{l}$ is expressed through the volume $V_{\mathrm{d}}$ (Fig. 1) and its basic linear sizes:

$$
V_{\mathrm{a}}^{l}=V_{\mathrm{d}} \frac{d^{2}}{D^{2}}\left(1-\frac{d}{2 l_{\mathrm{d}}}\right),
$$

where $d$ is the armature diameter, $D$ is the motor external diameter, $l_{\mathrm{d}}$ is the motor length.

With respect to the relative dimensionless sizes $x=\frac{d}{D}$, $y=\frac{l_{\mathrm{d}}}{D}:$

$$
V_{\mathrm{a}}^{l}=V_{\mathrm{d}} x^{2}\left(1-\frac{x}{2 y}\right) .
$$

As a result of substitution (11) in (9) mechanical work is defined by expression:

$$
A_{\mathrm{mec}}=V_{\mathrm{d}} x^{2}\left(1-\frac{x}{2 y}\right) \frac{B_{\mathrm{max}}^{2}}{2 \mathrm{~m}_{0}} \frac{\left(1-k_{\mathrm{a}}\right)^{2 n+1}}{(2 n+1)} .
$$

As it is recommended in [1-4], maximal work in unit volume can be achieved when the relative sizes $x$ and $y$ lay in rather wide limits:

$$
A_{\mathrm{mec}}=0.159 \cdot 10^{7} V_{\mathrm{d}} x^{2}\left(1-\frac{x}{2 y}\right) \frac{\left(1-k_{\mathrm{a}}\right)^{2 n+1}}{(2 n+1)}
$$

Therefore, $x$ can be in the range $0.41 \ldots 0.57$ and $y$ can be in the range $0.62 \ldots 1.2$. It is possible to explain rather wide range of change of these sizes by imperfection of used techniques of the specified parameters optimization.

If magnet weight is approximately equal to steel magnetic circuit weight, the weight of the motor can be defined as $m_{\mathrm{d}}=V_{\mathrm{d}} \Gamma_{\mathrm{st}}$ (where, $\Gamma_{\mathrm{st}}=7800 \mathrm{~kg} / \mathrm{m}^{3}$ ), that allows to derive the expression for specific energy in LEMM volume:

$$
a_{\mathrm{mec}}=\frac{A_{\mathrm{mec}}}{m_{\mathrm{d}}}=203.8 x^{2}\left(1-\frac{x}{2 y}\right) \frac{\left(1-k_{\mathrm{a}}\right)^{2 n+1}}{(2 n+1)} .
$$

Settlement values of the specific energy $a_{\text {mec }}$ depending on magnetic field increase degree and factor of armature penetration in the range of recommended $x$ and $y$ values are also stated in Fig. 3.

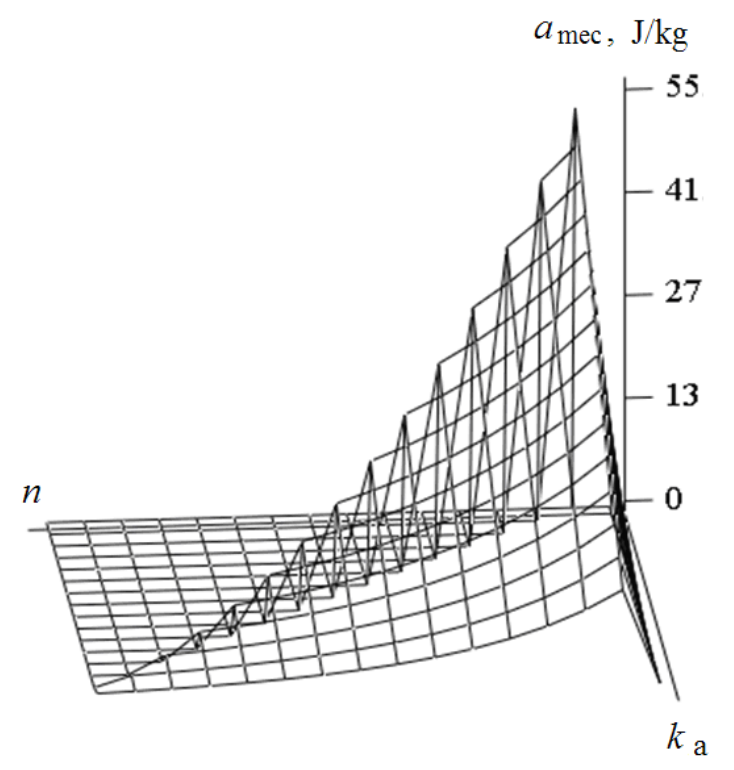

Fig. 3. The plot of dependence of specific energy in volume of the motor.

With respect to the kinetic energy balance equating (1) and (5):

$$
\frac{m_{\mathrm{a}} v^{2}}{2}=\frac{B_{\max }^{2} S_{\text {म }_{\max }\left(1-k_{\mathrm{a}}\right)^{(2 n+1)}}}{2 \mathrm{M}_{0}(2 n+1)} .
$$

Setting $m_{\mathrm{a}}=\gamma_{\mathrm{st}} V_{\mathrm{a}}$ :

$$
\frac{\Gamma_{\mathrm{st}} v^{2}}{2}=\frac{B_{\max }^{2}\left(1-k_{\mathrm{a}}\right)^{(2 n+1)}}{2 \mathrm{~m}_{0}(2 n+1)}
$$

where $\Gamma_{\text {st }}=7800 \mathrm{~kg} / \mathrm{m}^{3}$ is the steel density.

As it follows from (9) the armature speed at the end of energy conversion is:

$$
v=\sqrt{\frac{B_{\max }^{2}\left(1-k_{\mathrm{a}}\right)^{(2 n+1)}}{\mathrm{M}_{0} \Gamma_{\mathrm{st}}(2 n+1)}} .
$$

As it follows from the function (10), analysis the armature final speed when it is once accelerated in the longitudinal magnetic field depends only on the practical design characteristics $\Gamma_{\mathrm{st}}, B_{\max }$, penetration coefficient $k_{\mathrm{a}}$ describing relative depth and field change manner along the armature position coordinate. The dependence of the armature speed $v=f n, k_{\mathrm{a}}$ on induction change manner and initial armature penetration depth is shown in Fig. 4. 


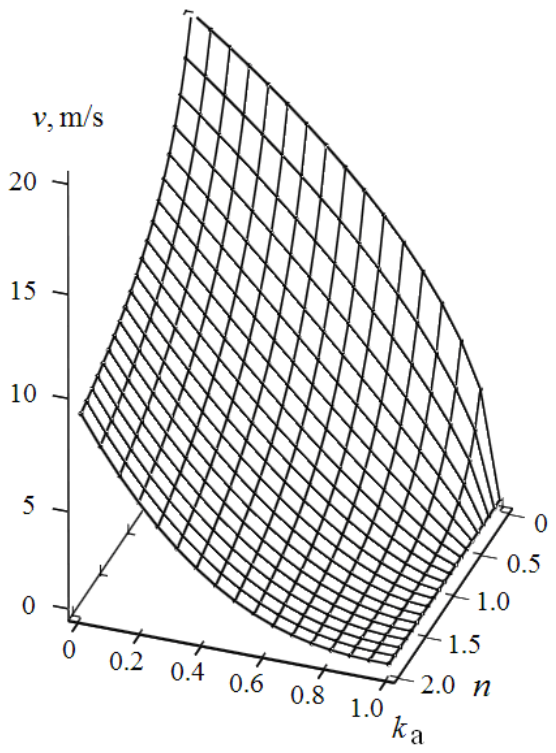

Fig. 4. The plot of $v=f n, k_{\mathrm{a}}$

If $B_{\max }=2.0 \mathrm{~T}$ for $n=0$ and $k_{\mathrm{a}}=0$ in (8), then:

$$
v_{\max }=\sqrt{\frac{B_{\max }^{2}}{\mathrm{M}_{0} \Gamma_{\mathrm{st}}}}=\sqrt{\frac{4}{4 \mathrm{p} 10^{-7} \cdot 7800}}=20 \mathrm{~m} / \mathrm{s} .
$$

If the induction dependence on the armature coordinate is close to real one then if, for example, $n=1$ (Fig. 4), the maximum speed is:

$$
v_{\max }=\sqrt{\frac{B_{\max }^{2}}{\mathrm{M}_{0} \Gamma_{\mathrm{st}} \cdot 3}}=\sqrt{\frac{4}{4 \mathrm{p} 10^{-7} \cdot 7800 \cdot 3}}=11.6 \mathrm{~m} / \mathrm{s} .
$$

\section{CONCLUSIONS}

At maximal increase of magnetic field $(n=0)$ and the minimal depth of penetration of the armature $k_{\mathrm{a}}=0$ specific energy in LEMM volume can reach $a_{\text {mec }}=54 \mathrm{~J} / \mathrm{kg}$. The value of a specific parameter of energy is certain at the greatest possible value of the armature displacement corresponding to the recommended ranges of the parameters $x$ and $y$.

The practically used electromechanical system with LEMM is characterized by quite certain factor of penetration of the armature. If the armature has two operating air gaps the armature penetration factor is in the range $k_{\mathrm{a}}=0.7 \ldots 0.8$. If $x=0.45$ and $y=1.0$, then the maximum energy in optimum volume can reach the values $a_{\text {mec }} \approx 12 \ldots 15 \mathrm{~J} / \mathrm{kg}$.

As it follows from the maximum armature speed $v_{\max }=20 \mathrm{~m} / \mathrm{s}$ a kind of magnetic field has no influence on the energy conversion process. The maximum speed at the end of the energy conversion process is determined only by magnetic core material practical design characteristics and magnetic properties, induction change character in the operating air gap and armature penetration initial depth.

\section{References}

[1] V.P. Pevchev, "The superexitation and efficiency relation in a shortstroke pulsed electromagnetic motor of a seismic source", Journal of Mining Science, vol. 46, Num. 6, pp. 656-665, 2010.

[2] K.M. Usanov, G.G. Ugarov, V.I. Moshkin, "Linear impulse electromagnetic drive of machines with self-contained power supplies", Kurgan: Kurgan University, p. 284, 2006.

[3] S.N. Kuchankov, E.M. Timoshenko, "Nonsteady thermal conditions of electromagnetic motors in shock-type equipment", Journal of Mining Science, vol. 34, Num.2., pp. 148-152, 1998.

[4] N.P. Ryashentsev, G.G. Ugarov, V.N. Fedonin, A.T. Malov, Electroprivod s lineynimi electromagnitnimi dvigatelyami, Novosibirsk, Nauka, p. 150, 1981.

[5] L.A. Neiman, V.Y. Neiman, "Linear synchronous electromagnetic machines for lowfrequency impact technologies", Russian Electrical Engineering, vol. 85, Num. 12, pp. 752-756, 2014.

[6] V.Y. Neiman, "Integrated linear electromagnetic motors for pulsed technologies", Russian Electrical Engineering, vol. 74, Num. 9, pp. 30 35, 2003.

[7] G.G. Ugarov, V.Y. Neiman, "Evaluation of operating conditions for electromagnetic impactors", Journal of Mining Science, vol. 32, Num. 4, pp. 305-312, 1996. 